\title{
Reliability of time dependent stress-strength system for various distributions
}

\author{
${ }^{1}$ N.Swathi , ${ }^{2}$ T.S.Uma Maheswari \\ ${ }^{1,2}$ Department of Mathematics, Kakatiya University, Warangal, A.P.
}

\begin{abstract}
Reliability analysis of time dependent stress strength system is carried out by considering each of stress variables are deterministic and strength variables are random - fixed and vice versa for different distributions .Reliability computations were done for different cycle lengths for different distributions. The number of cycles in any period of time ' $t$ ' is assumed to be deterministic, stress and strength follows Exponential distribution and Rayleigh distribution, it is observed that the reliability of the system is decreased when the number of cycles increased and system reliability rapidly change in Rayleigh distribution than the Exponential distribution. In the deterministic stress and random fixed strength and vice versa, for various parameter values, reliability is computed.
\end{abstract}

\section{Introdution}

Time dependent stress strength models by considering with the repeated application of stress and also considering the change of the distribution of strength with time. "Stress" is used to indicate any agency that tends to induce "failure", while "Strength" indicates any agency resisting "failure". "Failure is defined to have occurred when the actual stress exceed the actual strength.

There is uncertainty about the stress and strength random variables at any instant of time and also about the behavior of the random variables with respect to time and cycles. The two terms 'deterministic' and 'random fixed' are used to describe these two uncertainties .In deterministic, the variables assumes values that are exactly known a priori .Random fixed refers to the behavior of the variable with respect to time is fixed or the variable varies in time in a known manner. The failure of components under repeated stresses had been investigated primarily .Repeated stresses are characterized by the time, each load applied and the behavior of time intervals between the application of loads.

We prefer $R_{n}$ the reliability after $\mathrm{n}$ cycles, to $\mathrm{R}(\mathrm{t})$, the reliability at time $\mathrm{t}$, where $\mathrm{t}$ is continuous .Simply when cycle times are deterministically known $\mathrm{R}(\mathrm{t})=R_{n}, t_{n}<\mathrm{t} \leq t_{n+1}$, where $t_{i}$ is instant in time at which the $i^{\text {th }}$ cycle occurs. The time dependent load was discussed by several researchers. Some of them are Bilikam et al.,[1] ,Kechengshen[2] , M.N.Gopalan[3] and Dongshang chang[4].

In the present paper, we have discussed deterministic stress and random fixed strength and vice versa, we had take Exponential and Rayleigh distributions. Reliability computations were done for different cycle lengths. The result is that the system reliability rapidly changes in Rayleigh distribution than the Exponential distribution.

\section{Statistical Method}

$\mathrm{X}$ and $\mathrm{Y}$ denote the stress and strength of the system. $\mathrm{f}(\mathrm{X})$ and $\mathrm{g}(\mathrm{Y})$ are probability density functions of $\mathrm{X}$ and $\mathrm{Y}$. Then the reliability of the system is

$$
\mathrm{R}=\int_{-\infty}^{\infty} f(X)\left[\int_{X}^{\infty} g(Y) d Y\right] d X
$$

or

$$
\mathrm{R}=\int_{-\infty}^{\infty} g(Y)\left[\int_{-\infty}^{Y} f(X) d X\right] d Y
$$

In the reliability computations for deterministic cycle times we can take two cases

Case 1: Deterministic stress and random fixed strength

Let the stress be $x_{0}$, a constant and the strength on the $i^{\text {th }}$ cycle $Y_{i}$ given by

$$
Y_{i}=Y_{0}-a_{i}, \mathrm{i}=123
$$


Where $a_{i} \leq 0$ are known constants. Further, the $a_{i}$ 's are assumed non - decreasing in time. The p.d.f of $y_{0}, g_{0}\left(y_{0}\right)$ is assumed known. Then

$$
\begin{aligned}
p\left[E_{n}\right] & =p\left(x_{n}<=y_{n}\right) \\
& =\int_{x_{0}+a_{n}}^{\infty} g_{0}\left(y_{0}\right) d y_{0}
\end{aligned}
$$

All but the last term in the R.H.S of above equations are 1's because of restrictions on the $a_{i}$ 's which cause the strength $y_{i}$ to decrease in time. Hence

$$
R_{n}=p\left[E_{n}\right]=\int_{x_{0}+a_{n}}^{\infty} g_{0}\left(y_{0}\right) d y_{0}
$$

Case 2: Random fixed stress and deterministic strength

Let $X_{i}=X_{0}+b_{i}, \mathrm{i}=1,2,3$,--- denote the stress in cycle $\mathrm{i}$, where $b_{i}$ 's are known non negative constants, non decreasing in time. Further let the strength be held constant at $y_{0}$. The p.d.f. of $X_{0}, f_{0}\left(x_{0}\right)$ is assumed known.

The restrictions on $b_{i}$ 's guarantee non- decreasing. Stress, which in turn ensure that

$$
\begin{aligned}
R_{n} & =p\left[E_{n}\right] \\
& =p\left(X_{n}<=Y_{n}\right) \\
& =p\left(X_{0}+b_{n}<=y_{0}\right) \\
R_{n} & =\int_{0}^{y_{0}-b_{n}} f_{0}\left(x_{0}\right) d x_{0}
\end{aligned}
$$

\section{Reliability computations}

1. A random variable $X$ is said to have Exponential distribution with parameter $\lambda>0$ if its p.d.f. is given by

$$
\mathrm{f}(\mathrm{x}, \lambda)=\lambda e^{-\lambda x} \text { and }
$$

Similarly a random variable $\mathrm{Y}$ is said to have an Exponential distribution with parameter $\mu>0$ if its p.d.f. is given by

$$
\mathrm{g}(\mathrm{y}, \mu)=\mu e^{-\mu y}
$$

For deterministic stress and random fixed strength, the system reliability

$$
\begin{aligned}
& R(t)=R_{n}=p\left[E_{n}\right] \\
& =p\left(x_{n}<=y_{n}\right) \\
& =\int_{x_{0}+a_{n}}^{\infty} g_{0}\left(y_{0}\right) d y_{0} \\
& =\int_{x_{0}+a_{n}}^{\infty} \mu e^{-\mu y_{0}} d y_{0} \\
& =e^{-\mu\left(x_{0}+a_{n}\right)}
\end{aligned}
$$

For random fixed stress and deterministic strength, the reliability of the system is 


$$
\begin{aligned}
& R(t)=R_{n}=p\left[E_{n}\right] \\
& =p\left(x_{n}<=y_{n}\right) \\
& =\int_{0}^{y_{0}-b_{n}} f_{0}\left(x_{0}\right) d x_{0} \\
& =\int_{0}^{y_{0}-b_{n}} \lambda e^{-\lambda x_{0}} d x_{0} \\
& =1-e^{-\lambda\left(y_{0}-b_{n}\right)}
\end{aligned}
$$

2. A random variable $X$ is said to have a Rayleigh distribution with parameter $\lambda$ if its p.d.f. is given by

$$
\mathrm{f}(\mathrm{x}, \lambda)=\lambda \mathrm{x} e^{-x^{2} \lambda / 2} \text { and }
$$

Similarly a random variable $\mathrm{Y}$ is said to have a Rayleigh distribution with parameter $\mu$ if its p.d.f. is given by

$$
\mathrm{g}(\mathrm{y}, \mu)=\mu \mathrm{y} e^{-\mu y^{2} / 2}
$$

For deterministic stress and random fixed strength, the reliability of the system is

$$
\begin{aligned}
& R_{n}=\int_{x_{0}+a_{n}}^{\infty} g_{0}\left(y_{0}\right) d y_{0} \\
& =\int_{x_{0}+a_{n}}^{\infty} \mu y_{0} e^{-\mu y^{2} / 2} d y_{0} \\
& =e^{-\mu\left(x_{0}+a_{n}\right)^{2} / 2}
\end{aligned}
$$

$$
R_{n}=\int_{0}^{y_{0}-b_{n}} f_{0}\left(x_{0}\right) d x_{0}
$$

For random fixed stress and deterministic strength, the reliability of a system is $=\int_{0}^{y_{0}-b_{n}} \lambda x_{0} e^{-\lambda x_{0}^{2} / 2} d x_{0}$

$$
=1-e^{-\lambda\left(y_{0}-b_{n}\right)^{2} / 2}
$$

\section{Conclusion:}

In Exponential distribution if stress and strength follow for case 1 and case 2 if the Strength parameter value $(\mu)$, and constant value $\left(x_{0}\right)$ are fixed and we can increase the cycle lengths then reliability decreases. In the same way if the stress parameter value $(\lambda)$ and constant value $\left(y_{0}\right)$ are fixed and we increases the cycle lengths then reliability decreased. In Rayleigh distribution if stress and strength follow case 1 and case 2 and the parameters $(\lambda, \mu)$ and constants $(\mathrm{x}, \mathrm{y})$ are fixed and increases the cycle lengths then reliability decreases. All these computations we can observe that take the cycle lengths with more differences then reliability decreases more.

Table 1: Exponential Distribution can take case 1:

\begin{tabular}{|c|c|c|c|c|c|c|}
\hline & $\mathrm{a}$ & $\mathrm{R}$ & $\mathrm{a}$ & $\mathrm{R}$ & $\mathrm{a}$ & $\mathrm{R}$ \\
\hline$\mu=0.5$ & 0.1 & 0.860708 & 0.2 & 0.818731 & 0.5 & 0.704688 \\
\hline $\mathrm{x}=0.2$ & 0.2 & 0.818731 & 0.4 & 0.740818 & 1 & 0.548812 \\
\hline & 0.3 & 0.778801 & 0.6 & 0.67032 & 1.5 & 0.427415 \\
\hline & 0.4 & 0.740818 & 0.8 & 0.606531 & 2 & 0.332871 \\
\hline & 0.5 & 0.704688 & 1 & 0.548812 & 2.5 & 0.25924 \\
\hline & 0.6 & 0.67032 & 1.2 & 0.496585 & 3 & 0.201897 \\
\hline & 0.7 & 0.637628 & 1.4 & 0.449329 & 3.5 & 0.157237 \\
\hline & 0.8 & 0.606531 & 1.6 & 0.40657 & 4 & 0.122456 \\
\hline & 0.9 & 0.57695 & 1.8 & 0.367879 & 4.5 & 0.095369 \\
\hline & 1 & 0.548812 & 2 & 0.332871 & 5 & 0.074274 \\
\hline
\end{tabular}


Reliability of time dependent stress-strength system for various distributions Table 2:

\begin{tabular}{|c|c|c|c|c|}
\hline & $\mathrm{a}$ & $\mathrm{R}$ & $\mathrm{a}$ & $\mathrm{R}$ \\
\hline$\mu=0.2$ & 0.1 & 0.802519 & 1 & 0.67032 \\
\hline $\mathrm{X}=1$ & 0.2 & 0.786628 & 1.2 & 0.644036 \\
\hline & 0.3 & 0.771052 & 1.4 & 0.618783 \\
\hline & 0.4 & 0.755784 & 1.6 & 0.594521 \\
\hline & 0.5 & 0.740818 & 1.8 & 0.571209 \\
\hline & 0.6 & 0.726149 & 2 & 0.548812 \\
\hline & 0.7 & 0.71177 & 2.2 & 0.527292 \\
\hline & 0.8 & 0.697676 & 2.4 & 0.506617 \\
\hline & 0.9 & 0.683861 & 2.6 & 0.486752 \\
\hline & 1 & 0.67032 & 2.8 & 0.467666 \\
\hline
\end{tabular}

Table 3:

\begin{tabular}{|c|c|c|c|c|}
\hline & $\mathrm{a}$ & $\mathrm{R}$ & $\mathrm{a}$ & $\mathrm{R}$ \\
\hline$\mu=0.2$ & 0.1 & 0.960789 & 1 & 0.802519 \\
\hline $\mathrm{X}=0.1$ & 0.2 & 0.941765 & 2 & 0.657047 \\
\hline & 0.3 & 0.923116 & 3 & 0.537944 \\
\hline & 0.4 & 0.904837 & 4 & 0.440432 \\
\hline & 0.5 & 0.88692 & 5 & 0.360595 \\
\hline & 0.6 & 0.869358 & 6 & 0.29523 \\
\hline & 0.7 & 0.852144 & 7 & 0.241714 \\
\hline & 0.8 & 0.83527 & 8 & 0.197899 \\
\hline & 0.9 & 0.818731 & 9 & 0.162026 \\
\hline & 1 & 0.802519 & 10 & 0.132655 \\
\hline
\end{tabular}

Table4: Exponentialdistributioncantakecase2:

\begin{tabular}{|c|c|c|c|c|c|c|}
\hline & $b$ & $R$ & $b$ & $R$ & $b$ & $R$ \\
\hline$\lambda=0.5$ & 0.1 & 0.913706 & 0.2 & 0.909282 & 0.5 & 0.894601 \\
\hline$y=5$ & 0.2 & 0.909282 & 0.4 & 0.899741 & 1 & 0.864665 \\
\hline & 0.3 & 0.904631 & 0.6 & 0.889197 & 1.5 & 0.826226 \\
\hline & 0.4 & 0.899741 & 0.8 & 0.877544 & 2 & 0.77687 \\
\hline & 0.5 & 0.894601 & 1 & 0.864665 & 2.5 & 0.713495 \\
\hline & 0.6 & 0.889197 & 1.2 & 0.850431 & 3 & 0.632121 \\
\hline & 0.7 & 0.883516 & 1.4 & 0.834701 & 3.5 & 0.527633 \\
\hline & 0.8 & 0.877544 & 1.6 & 0.817316 & 4 & 0.393469 \\
\hline & 0.9 & 0.871265 & 1.8 & 0.798103 & 4.5 & 0.221199 \\
\hline & 1 & 0.864665 & 2 & 0.77687 & 5 & 0 \\
\hline
\end{tabular}

Table 5:

\begin{tabular}{|c|c|c|c|c|}
\hline & $\mathrm{b}$ & $\mathrm{R}$ & $\mathrm{b}$ & $\mathrm{R}$ \\
\hline$\lambda=1$ & 0.1 & 0.850431 & 0.2 & 0.834701 \\
\hline $\mathrm{y}=2$ & 0.2 & 0.834701 & 0.4 & 0.798103 \\
\hline & 0.3 & 0.817316 & 0.6 & 0.753403 \\
\hline & 0.4 & 0.798103 & 0.8 & 0.698806 \\
\hline & 0.5 & 0.77687 & 1 & 0.632121 \\
\hline & 0.6 & 0.753403 & 1.2 & 0.550671 \\
\hline & 0.7 & 0.727468 & 1.4 & 0.451188 \\
\hline & 0.8 & 0.698806 & 1.6 & 0.32968 \\
\hline & 0.9 & 0.667129 & 1.8 & 0.181269 \\
\hline & 1 & 0.632121 & 2 & 0 \\
\hline
\end{tabular}

Table 6: Rayleigh distribution can take case 1: 
Reliability of time dependent stress-strength system for various distributions

\begin{tabular}{|c|c|c|c|c|c|c|}
\hline & $\mathrm{a}$ & $\mathrm{R}$ & $\mathrm{a}$ & $\mathrm{R}$ & $\mathrm{a}$ & $\mathrm{R}$ \\
\hline$\mu=0.5$ & 0.1 & 0.977751 & 0.2 & 0.960789 & 0.5 & 0.884706 \\
\hline 0.2 & 0.2 & 0.960789 & 0.4 & 0.913931 & 1 & 0.697676 \\
\hline & 0.3 & 0.939413 & 0.6 & 0.852144 & 1.5 & 0.485537 \\
\hline & 0.4 & 0.913931 & 0.8 & 0.778801 & 2 & 0.298197 \\
\hline & 0.5 & 0.884706 & 1 & 0.697676 & 2.5 & 0.161621 \\
\hline & 0.6 & 0.852144 & 1.2 & 0.612626 & 3 & 0.077305 \\
\hline & 0.7 & 0.816686 & 1.4 & 0.527292 & 3.5 & 0.032631 \\
\hline & 0.8 & 0.778801 & 1.6 & 0.444858 & 4 & 0.012155 \\
\hline & 0.9 & 0.738968 & 1.8 & 0.367879 & 4.5 & 0.003996 \\
\hline & 1 & 0.697676 & 2 & 0.298197 & 5 & 0.001159 \\
\hline
\end{tabular}

Table 7 : Rayleigh distribution can take case 2

\begin{tabular}{|c|c|c|c|c|c|c|}
\hline & $\mathrm{b}$ & $\mathrm{R}$ & $\mathrm{b}$ & $\mathrm{R}$ & $\mathrm{b}$ & $\mathrm{R}$ \\
\hline$\lambda=0.5$ & 0.1 & 0.997527 & 0.2 & 0.996849 & 0.5 & 0.99367 \\
\hline $\mathrm{y}=5$ & 0.2 & 0.996849 & 0.4 & 0.994958 & 1 & 0.981684 \\
\hline & 0.3 & 0.996004 & 0.6 & 0.992093 & 1.5 & 0.953229 \\
\hline & 0.4 & 0.994958 & 0.8 & 0.987845 & 2 & 0.894601 \\
\hline & 0.5 & 0.99367 & 1 & 0.981684 & 2.5 & 0.790389 \\
\hline & 0.6 & 0.992093 & 1.2 & 0.972948 & 3 & 0.632121 \\
\hline & 0.7 & 0.990172 & 1.4 & 0.960836 & 3.5 & 0.430217 \\
\hline & 0.8 & 0.987845 & 1.6 & 0.944424 & 4 & 0.221199 \\
\hline & 0.9 & 0.985042 & 1.8 & 0.922695 & 4.5 & 0.060587 \\
\hline & 1 & 0.981684 & 2 & 0.894601 & 5 & 0 \\
\hline
\end{tabular}

Figure 1:

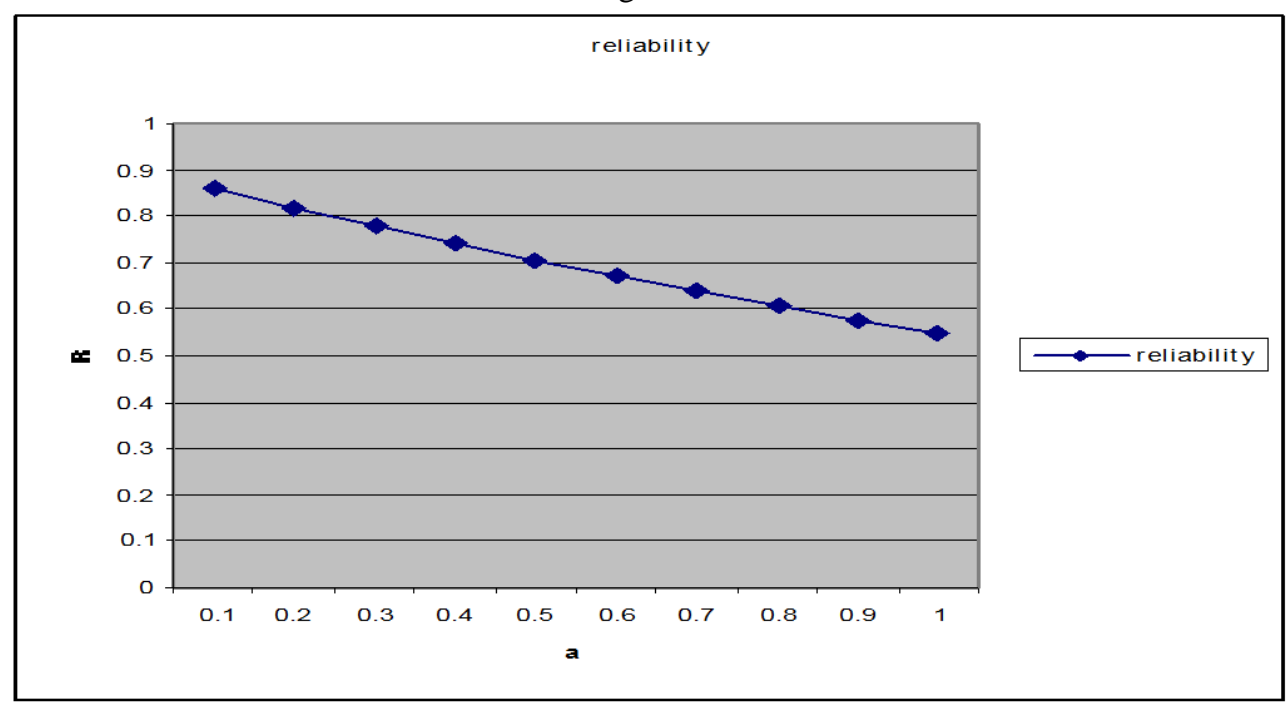



Figure 2:

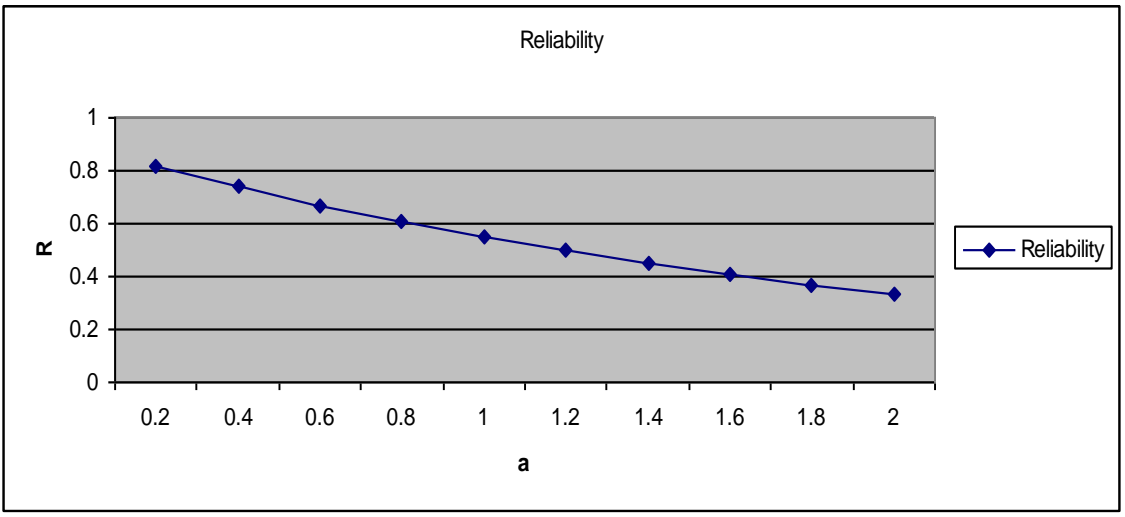

Figure3 3

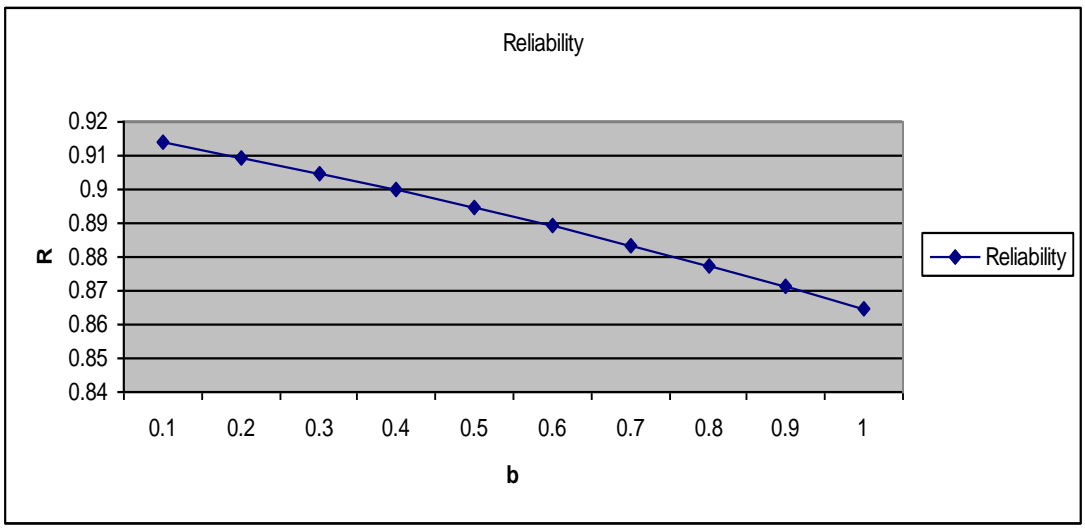

Figure 4:

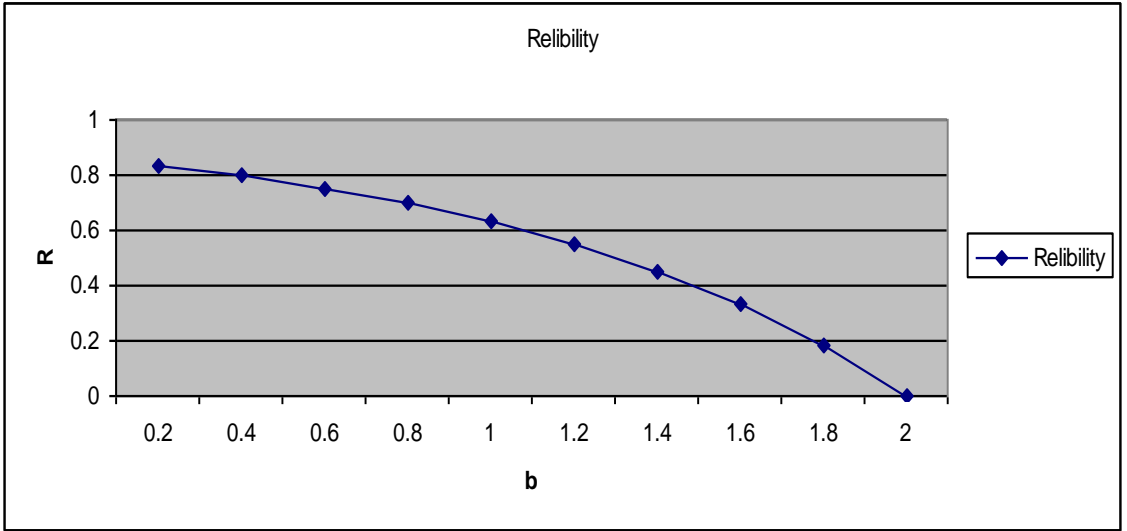

Figure5 5

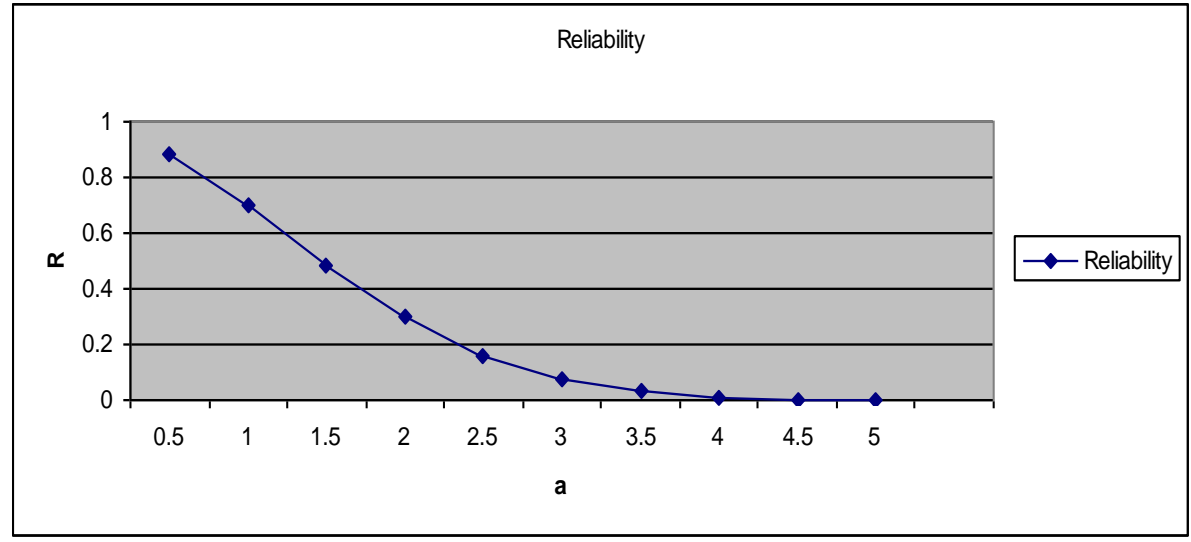




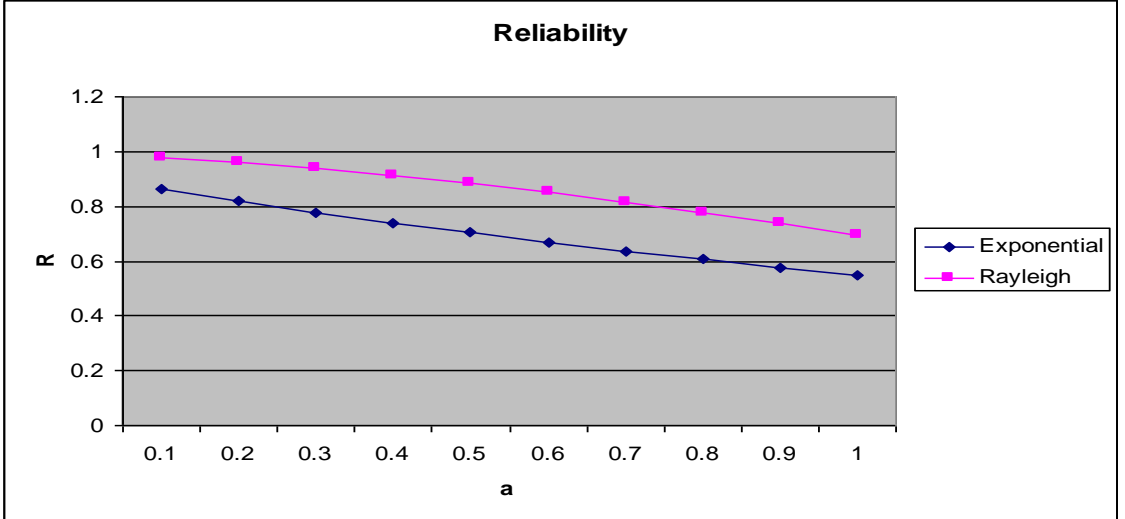

\section{References:}

[1] Bilikam, J.Edward(1985) : Some stochastic Stress- Strength processes , vol .R-34 , pp: 269-274

[2] Kecheng Shen(1988) : On the relation between component failure rate and stree - strength distributional charecterstics , Micro Electronics Reliability, vol. 28 , pp:801-812.

[3] M.N.Gopalan and P.Venkateswarlu(1982) : reliability analysis of time dependent cascade system with deterministic cycle times , Micro Electronics Reliability, vol. 22, pp:841-872.

[4] Dong Shang Chang (1995) : Reliability bounds for the stress-strength model, vol.29, pp:15-19.

[5] Kapur,K.C. and L.R.Lamberson(1977) : Reliability in Engineering Design, Jhon Wiley and sons, Inc., New York.

[6] S.C.Gupta and V.K.Kapoor : Fundamentals of Mathematical Statistics.

[7] R.P.S.Yadav(1973) : A Reliability Model for stress strength problem, Micro Electronics Reliability, vol.12, pp:119-123.

[8] M.N.Gopalan and P.Venkateswarlu(1983) : Reliability analysis of time dependent cascade system with random cycle times, vol. 23, pp:355-366. 